Monatsschr Kinderheilkd 2022 · 170:443-452 https://doi.org/10.1007/s00112-020-01116-2 Angenommen: 10. Dezember 2020

Online publiziert: 15. Januar 2021

๑ Der/die Autor(en) 2021

\section{Redaktion}

B. Koletzko, München

T. Lücke, Bochum

E. Mayatepek, Düsseldorf

N. Wagner, Aachen

S. Wirth, Wuppertal

F. Zepp, Mainz

\author{
Johannes Hofer ${ }^{1,2,3}$ • Johannes Fellinger ${ }^{1,2,4}$ \\ ${ }^{1}$ Institut für Sinnes und Sprachneurologie, Konventhospital Barmherzige Brüder Linz, Linz, Österreich \\ ${ }^{2}$ Research Institute for Developmental Medicine, Johannes Kepler Universität Linz, Linz, Österreich \\ ${ }^{3}$ Department für Pädiatrie I, Medizinische Universität Innsbruck, Innsbruck, Österreich \\ ${ }^{4}$ Abteilung für Sozialpsychiatrie der Universitätsklinik für Psychiatrie und Psychotherapie, Medizinische \\ Universität Wien, Wien, Österreich
}

\title{
Autismus-Spektrum-Störungen: von der Früherfassung zu Intervention und Begleitung
}

\section{Hintergrund}

Autismus-Spektrum-Störungen (ASS) zeichnen sich durch ihre Heterogenität aus, gleichen sich aber in ihrem phänotypischen Kern: der Beeinträchtigung der Entwicklungsprozesse, welche die soziale Interaktion und Wechselseitigkeit begründen.

Die Prävalenz von ASS liegt bei 0,9-1,1\% [1]. Bezüglich des Geschlechterverhältnisses überwiegt mit 3-4:1 das männliche Geschlecht. Weibliche Betroffene scheinen häufiger unerkannt $\mathrm{zu}$ bleiben und später diagnostiziert zu werden [2]. Die Prävalenz von ASS bei Geschwisterkindern liegt bei $10-20 \%$ [3].

Die genaue ätiologische Grundlage von ASS ist ungeklärt, wenngleich 74-93\% des ASS-Risikos genetisch bedingt sind [4]. Es wurden bisher keine Genveränderungen gefunden, welche ein spezifisches ASS-Risiko bedingen, ohne einen mindestens ebenso großen Risikoanteil bezüglich weiterer Entwicklungsstörungen oder Epilepsie mitzutragen [5].

Belegte, nichtgenetische Risikofaktoren sind u. a. erhöhtes Konzeptionsalter (Mutter $\geq 40$ LJ; Vater $\geq 50$ LJ), Valproatexposition in der Schwangerschaft sowie Frühgeburtlichkeit $<32$ SSW. Impfungen ließen sich als Risikofaktoren ausschließen [6].

Im Vergleich zu "neurotypischen“ Kindern weisen Kinder mit ASS ein akzeleriertes ZNS-Wachstum mit verän- derter Konnektivität auf. Dies könnte im Zusammenhang stehen, mit den gehäuft unspezifisch auffälligen EEG-Befunden [7].

\section{Klassifikation}

Die in der ICD-10 (• Tab. 1) gewählte kategoriale Einteilung tiefgreifender Entwicklungsstörungen lässt sich weder auf klinischer, neurobiologischer noch kognitiver Ebene als stabil bewerten [8]. International wird überwiegend die DSM5-Klassifikation, welche auch in der ICD11 Entsprechung finden wird, verwendet. Diese fasst dimensional die unterschiedlichen Autismuskategorien unter dem Begriff ASS zusammen und sieht eine beschreibende Spezifizierung bezüglich kognitiver und sprachlicher Fertigkeiten vor (• Tab. 1). Das in der ICD-10 als eigene Kategorie geführte Asperger-Syn-

Infobox 1 Weitere Informationen zum Thema online

Bundesverband Autismus Deutschland: www. autismus.de

Österreichische Autistenhilfe: www.

autistenhilfe.at

M-CHAT Übersetzungen zum Download: https://mchatscreen.com

Beispiel für internationale Autismusselbsthilfe: www.autismspeaks.org AWMF-S3-Leitlinie Autismus Diagnostik: www.awmf.org

AWMF-S3-Leitlinie Therapie in Arbeit: www. awmf.org/leitlinien/detail/anmeldung/1/II/ 028-047.html 


\begin{tabular}{ll}
\hline Kategorien & F84.0 Frühkindlicher Autismus \\
F84.1 Atypischer Autismus \\
F84.2 Rett-Syndrom \\
F84.3 Desintegrative Störung des Kindesalters \\
F84.4 Überaktive Störung mit Intelligenzminderung und Bewegungs- \\
stereotypien \\
F84.5 Asperger-Syndrom \\
F84.8 Sonstige tiefgreifende Entwicklungsstörungen \\
F84.9 Tiefgreifende Entwicklungsstörung nicht näher bezeichnet \\
1) Wechselseitige soziale Interaktion \\
2) Qualitative Beeinträchtigung der Kommunikation \\
Kerndomänen Stereotype Verhaltensweisen und Sonderinteressen \\
Kriterien der Domä- \\
nen \\
Domë Zur Regulation sozialer Interaktion eingesetzte/eingesetzter Blick- \\
kontakt, Mimik, Körperhaltung und Gestik sind beeinträchtigt \\
b) Unfähigkeit, altersgemäße Beziehungen zu Gleichaltrigen aufzu- \\
nehmen \\
c) Mangel an sozioemotionaler Gegenseitigkeit \\
d) Mangel, spontan Freude, Interessen oder Tätigkeiten zu teilen \\
Domäne 2 ( $\geq 1$ der folgenden 4 Kriterien treffen zu): \\
a) Entwicklungsstörung der gesprochenen Sprache ohne Kompensati- \\
on durch Gestik/Mimik \\
b) Relative Unfähigkeit, eine Konversation zu beginnen/erhalten \\
c) Stereotype, repetitive oder eigentümliche Verwendung von Spra- \\
che \\
d) Mangel an spontanem „Als-ob-Spiel“ bzw. Interaktionsspielen \\
Domäne 3 ( $\geq 1$ der folgenden 4 Kriterien treffen zu) \\
a) Intensive Beschäftigung mit stereotypen/begrenzten Interessen \\
b) Spezifische, nichtfunktionale Handlungen oder Rituale \\
c) Stereotype und repetitive motorische Manierismen \\
d) Durchgängige Beschäftigung mit Teilaspekten bzw. nichtfunktio- \\
nalen Elementen von Gegenständen \\
Domäne 1+ 2+ 3: In Summe müssen $\geq 6$ Kriterien erfüllt sein
\end{tabular}

Symptombeginn Vor dem Alter von 3 Jahren

Schweregrade Indirekt über die Kategorien

Kognition Über Achsenkodierung (Achse III)

Sprache Über Achsenkodierung (Achse II)

Ätiologie Über Achsenkodierung (Achsen IV, V); Rett-Syndrom unter F84.2 als eigenständige psychiatrische Diagnose

Komorbiditäten Kodierung auf der entsprechenden Achse (gewisse Entitäten aber als Ausschlussdiagnose z. B.: ADHS)
299.00 Autismus-Spektrum-Störung

A) Defizite der sozialen Kommunikation und Interaktion

B) Restriktive, repetitive Verhaltensweisen, Interessen und Aktivitäten

Domäne A (alle 3 Kriterien treffen zu)

a) Defizite der sozial-emotionalen Reziprozität

b) Defizite der nonverbalen Kommunikation

c) Defizite in Entwicklung, Erhalt und Verständnis von Freundschaften

Domäne $B$ ( $\geq 2$ der folgenden 4 Kriterien treffen zu) a) Stereotype Verhaltensweisen (motorisch, sprachlich, spielerisch)

b) Beharren auf Routinen

c) Eingeschränkte/intensive Interessen

d) Hyper-/Hyporeaktivität auf sensorische Reize; sensorische Interessen

Zusätzliche Forderungen (alle 3 treffen zu)

- Domäne C: Symptome müssen in früher Kindheit vorhanden sein. Sie können sich erst bei entsprechend hohen sozialen Anforderungen manifestieren

- Domäne D: Symptome müssen zu klinisch bedeutsamer Behinderung im Alltag führen (sozial, schulisch, beruflich)

- Domäne E: Symptome lassen sich nicht durch intellektuelle Behinderung oder globale Entwicklungsstörung erklären

Symptome sind in der frühen Kindheit vorhanden, können sich aber auch erst dann voll manifestieren, wenn die sozialen Anforderungen steigen

Je 3 Schweregrade für Domäne $A$ und $B$ :

- Grad 1: benötigt Unterstützung

- Grad 2: benötigt beträchtliche Unterstützung

- Grad 3: benötigt sehr beträchtliche Unterstützung

Angabe mit/ohne geistige Beeinträchtigung

Angabe mit/ohne Sprachstörung

Zusätzliche Kodierung medizinischer oder genetischer Erkrankungen (Rett-Syndrom als genetische Kategorie); Angabe möglicher Umweltfaktoren

Zusätzliche Kodierung von anderen neurogenen Entwicklungsstörungen, psychischen Erkrankungen oder Verhaltensstörungen z. B. auch von ADHS drom, charakterisiert durch zumindest durchschnittliche Intelligenz und unauffällige formale Sprachentwicklung, findet sich im DSM-5 so nicht mehr (- Tab. 1).

\section{Kernsymptome}

Als herausragendes ASS-Kernsymptom ist die eingeschränkte Qualität/Quantität der sozialen Kommunikation und Interaktion zu verstehen: Es besteht im Vergleich zum kognitiven Entwicklungsniveau ein Defizit in der wechselseitigen Kommunikation und sozialen Interakti- on. Die sozioemotionale Reziprozität ist auffällig (Imitieren, Aufbau von Blickkontakt, soziales Lächeln, geteilte Aufmerksamkeit, Verständnis von Distanz und Nähe).

Die Fähigkeit zu und das Verständnis von nonverbaler Kommunikation sind eingeschränkt. Es zeigen sich Schwierigkeiten in der situationsadäquaten Ver- 
wendung und Interpretation von „kommunikativem“ Blickkontakt, Gestik, Mimik, Intonation und Körpersprache.

Auch die pragmatische Verwendung von Sprache, ihr Einsatz zum zwischenmenschlichen Austausch, das Verständnis sprachlich-kommunikativer Konventionen wie "turn-taking“, das sich sprachlich An-das-Gegenüber-Anpassen sowie das Nutzen von Intonation gelingen nur eingeschränkt.

Die soziale Kognition und damit die Fähigkeit zum Aufbau und zum Erhalt von Freundschaften und Beziehungen sind beeinträchtigt.

Für die Diagnose einer ASS wird auch das Vorliegen von restriktiven und repetitiven Verhaltensweisen, Interessen und Aktivitäten gefordert. Stereotype und repetitive Verhaltensweisen können sich sowohl motorisch, sprachlich als auch im Spielverhalten zeigen. Motorische Manierismen (z. B.: Hände wedeln, Körper wippen) finden sich bei ASS, aber auch bei Intelligenzminderung und/oder sensorischen Beeinträchtigungen.

Nichtfunktionale Verwendung unterschiedlicher Gegenstände im Sinne von Aufreihen nach bestimmten Mustern sowie repetitives Spiel nach exakten Ritualen und immer gleichen Abläufen sind häufig beobachtbare Symptome.

Kognitive Rigidität mit ihrem Ausdruck im Bestehen auf Routinen bzw. Nichttolerieren von Veränderung kann sich auf das Leben der ganzen Familie stark auswirken.

Eingeschränkte oder auch besonders intensive Interessen schränken den Alltag mit seinen Lernmomenten zusätzlich ein.

Sensorische Hypo- und/oder Hyperreaktivität können/kann die Emotionsregulation und Kooperationsfähigkeit beeinflussen.

\section{ASS-Symptome über die Lebensspanne}

Veränderungen des klinischen Erscheinungsbildes sind über die Lebenspanne beträchtlich (•Abb. 1): Knapp zwei Drittel der betroffenen Kinder präsentieren sich mit ASS-typischen Symptomen bereits vor dem vollendeten zweiten Lebensjahr (•Tab. 2). Bei zumindest ei-

Monatsschr Kinderheilkd $2022 \cdot$ 170:443-452 https://doi.org/10.1007/s00112-020-01116-2 (c) Der/die Autor(en) 2021

\section{J. Hofer · J. Fellinger}

\section{Autismus-Spektrum-Störungen: von der Früherfassung zu Intervention und Begleitung}

\section{Zusammenfassung}

Autismus-Spektrum-Störungen zeigen eine Prävalenz von $1 \%$. Die unterschiedlich stark ausgeprägte, soziale Interaktions- und Kommunikationsstörung ist gekennzeichnet durch eingeschränkte Fähigkeit, sich sprachlich und nichtsprachlich auf andere Menschen einzustellen. Weitere Kernsymptome sind repetitive und stereotype Verhaltens- und Interessenmuster.

Symptome einer Autismus-Spektrum-Störung zeigen sich überwiegend bereits während des Kleinkindalters und persistieren meist über die Lebensspanne in unterschiedlicher Phänomenologie und unterschiedlichem Ausprägungsgrad.

Wichtige abzugrenzende, aber auch begleitende Störungen bei jungen Kindern sind: kognitive Entwicklungsstörungen, Aufmerk- samkeitsdefizit- und Hyperaktivitätssyndrom und Sprachstörungen.

Früherkennung ist häufig bereits im zweiten Lebensjahr möglich. Evidenzbasierte, effektive Frühinterventionsmodelle existieren, sind aktuell aber im deutschsprachigen Raum noch nicht flächendeckend verfügbar. Angesichts der hohen Belastung für Familie und Umfeld ist ein kompetentes ärztliches Case Management mit Vernetzung zu Systempartnern aus Bildungs- und Sozialwesen notwendig.

Schlüsselwörter

Entwicklungsdiagnostik · Entwicklungsstörung · Autismusscreening . Autismusintervention • "Medical home"

\section{Autism spectrum disorders: from early identification to intervention and case management}

\section{Abstract}

Autism spectrum disorders (ASD) are heterogeneous conditions with varying presentations and a prevalence of around $1 \%$. They are characterized by social communication deficits and repetitive sensory motor behavior.

The symptoms are present in the early developmental period and persist with varying phenomenology and severity over the lifespan. The most important differential diagnosis and comorbidities are intellectual developmental disorders, attention deficit hyperactivity disorder and developmental language disorders.
Early detection during the second year of life is possible. Evidence-based early intervention programs exist but are currently only locally available in German speaking countries. The burden of ASD for the family and the social environment is often tremendous. A competent medical case management in a network including partners from education and social welfare is crucial.

\section{Keywords}

Developmental assessment · Developmental disorder · Autism screening - Autism intervention $\cdot$ Medical home nem Viertel zeigt sich eine kommunikative Regression zwischen dem 18. und 24. Lebensmonat [9].

Im Kindergartenalter führen zunehmend zutage tretende Symptome wie mangelndes Interesse an der Interaktion mit anderen (Wahrnehmung als „angenehm" braves Kind, das sich selbst beschäftigt), fehlende Empathie, restriktive Interessen und ausgeprägte Stressreaktionen zur Abklärung. Im Schulalter sind Symptome wie Überfokussierung auf Lieblingsthemen, eingeschränkte so- ziale Fertigkeiten, Ängstlichkeit sowie Vermeidungsverhalten bezüglich sozialer Interaktion Hinweise. Insbesondere bei kognitiv gut begabten Kindern mit ASS bleibt eine Diagnosestellung oft lange aus.

Betroffene Erwachsene begeben sich nicht selten wegen eines zunehmenden Gefühls des Andersseins und des Nichtdazugehörens auf die Suche nach Erklärung und Hilfestellung. 


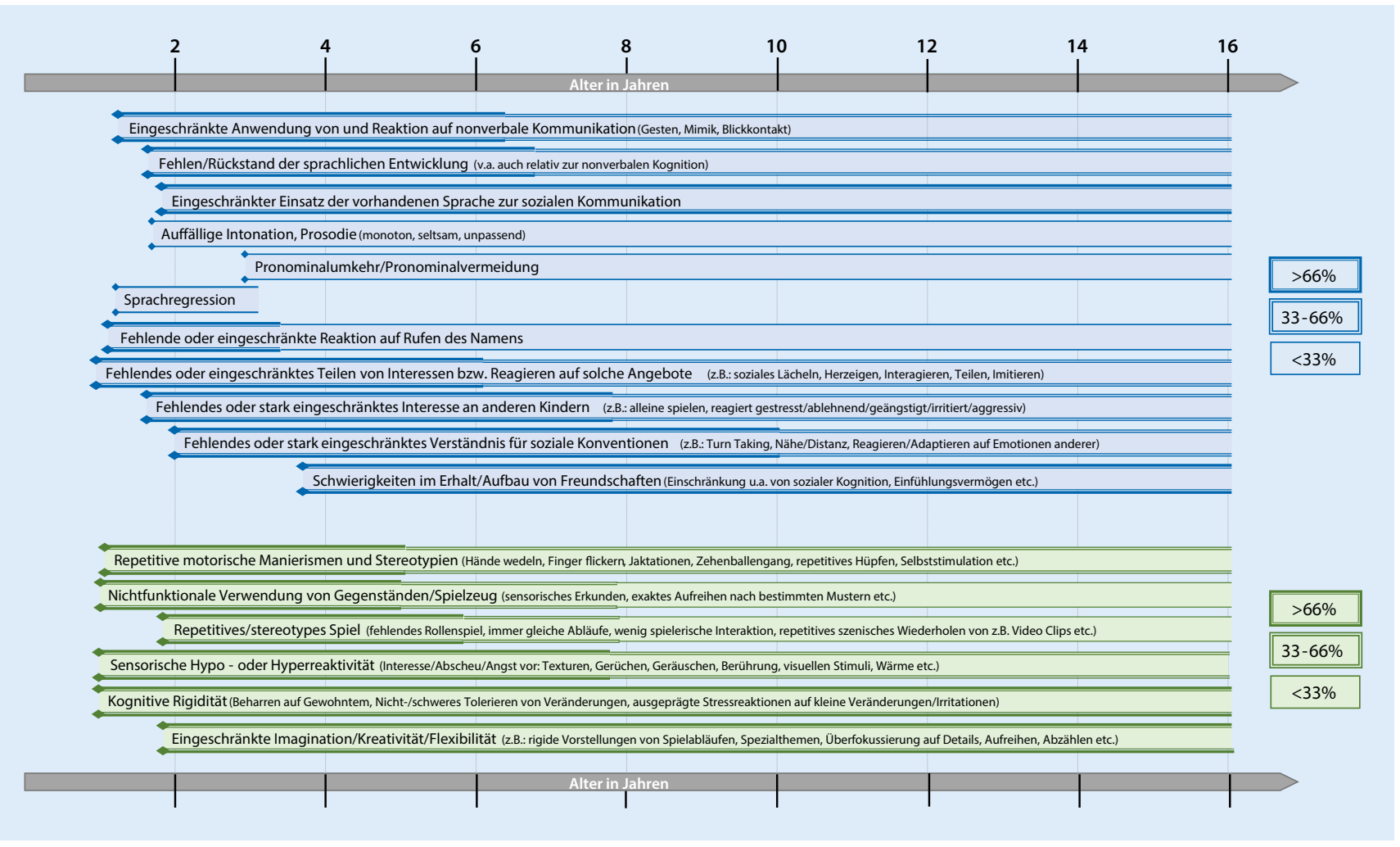

Abb. 1 A Prävalenz von Symptomen im Entwicklungsverlauf. Dicke der einhüllenden Linien repräsentiert die Häufigkeit. Blau umrandet: Kommunikation und soziale Interaktion. Grün: repetitive und stereotype Verhaltensmuster und Interessen $[6,7,10$, $13,14,30,31]$

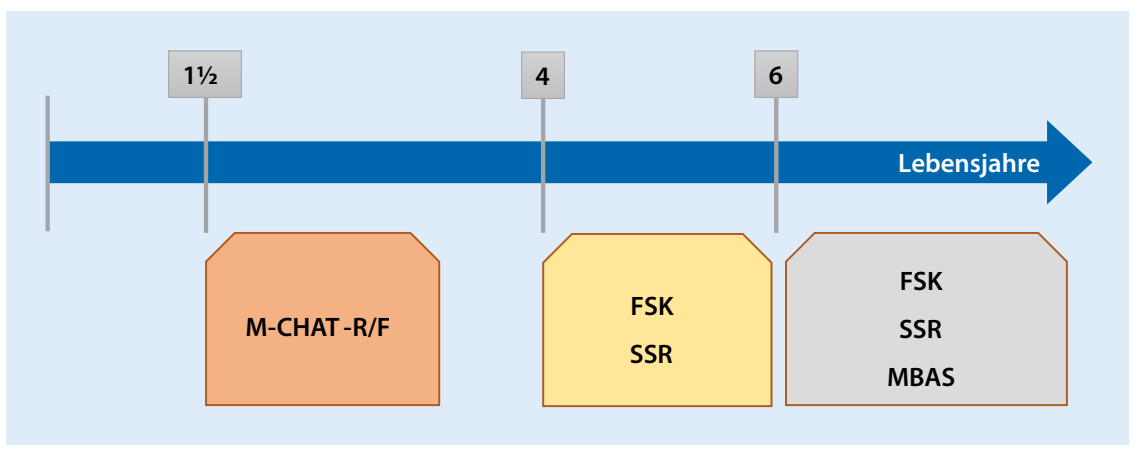

Abb. 2 × Laut AWMF-Leitlinie empfohlene Screening-Tools, bezogen auf das Alter bei Screening. M-CHAT-R/FModified Checklist for Autism in Toddlers Revised with Follow up questions, FSK Fragebogen zur Sozialen Kommunikation, SRSSkala für Soziale Responsivität, MBASMarburger Beurteilungsskala zum Asperger-Syndrom. (Freitag und VIlasaliu [6])

\section{Diagnosestabilität und Prognose von ASS}

ASS-Kernsymptome finden sich in den meisten Fällen in unterschiedlicher Ausprägung über den Lebenszeitverlauf, allerdings erfüllen bis zu $20 \%$ der Kinder, welche vor dem 3. Lebensjahr eine ASS-Diagnose erhalten, später die Diagnosekriterien nicht mehr. In diesen Fällen demarkieren sich dann meist andere Entwicklungsstörungen (Sprachentwicklungsstörungen, ADHS; [7]).

Die beste Prognose über die Lebensspanne haben ASS-betroffene Kinder, bei denen nach initialer Sprachverzögerung eine rasche Aufholentwicklung bis zum 3. Lebensjahr gegeben ist („bloomers“; [10]). Vor allem sprachliche und kognitive Fertigkeiten stellen Prädiktoren für das sozial-adaptive Outcome im Erwachsenenalter dar [11].
Bis zu ein Drittel der Kinder mit ASS zeigt sich als Erwachsene funktionell nahezu nonverbal, ohne dass dies durch die häufig assoziierte kognitive Beeinträchtigung ausreichend erklärbar wäre [12].

\section{Erfassung und Diagnosestellung}

Allein auf Basis des klinischen Eindrucks werden drei Viertel der von ASS betroffenen Kinder nicht rechtzeitig erkannt [13]. Zur frühen Erfassung können Screeningbemühungen beitragen. In der AWMFS3-Leitlinie [6] zur Diagnostik von ASS wird derzeit keine Empfehlung für ein flächendeckendes, jedoch für ein verdachtsorientiertes Screening ausgegeben (॰ Abb. 2).

\section{ASS-Diagnostik}

Nach einem positiven Screeningbefund bzw. bestehendem Verdacht hat eine spezialisierte diagnostische Abklärung zu erfolgen (• Abb. 3). Für die Einleitung die- 
Tab. 2 ASS-Frühsymptome [6, 7, 13]

Frühsymptome („red flags“) der ASS

Kein/seltenes soziales Lächeln

Ab 9 LM

Kein/seltenes Folgen des Blicks eines Gegenübers und Reagieren auf Zeigegesten

Keine/seltene Aufnahme des Blickkontakts (über 1s)

Ab 12 LM

Kein spontanes Teilen von Gefühlen oder keine Mitteilung von

Affekten

Keine/seltene Reaktion auf den eigenen Namen

Ab 12 LM

Ab 12 LM

Ab 12 LM

Stereotype Verhaltensweisen (z. B. Jaktationen, Manierismen, Drehen von Gegenständen)

Sensorische Auffälligkeiten (auditive/visuelle/olfaktorische/taktile Hypersensitivität)

Kein/seltenes protodeklaratives Zeigen mit dem Zeigefinger (um auf etwas hinzuweisen und Interesse zu teilen)

Kein/wenig Interesse an anderen Kindern

Ab 12 LM

Ab 12 LM

Ab 14 LM

Ab 14 LM

Jeglicher Verlust von Sprache!

Ab 14 LM

Fehlendes Rollenspiel (Füttern von Stofftier, Puppe zu Bett bringen, Ab 18 LM Spielfigur in Spielauto setzen)

Frühsymptome einer ASS mit normaler formaler Sprache und Kognition (z. B.: Asperger)

Kein, wenig oder inadäquater Kontakt (spielt bevorzugt alleine)

Stereotype Verhaltensweisen und Sprachgebrauch

Veränderungsängste sowie zwanghafte und ritualisierte Verhaltens weisen

Sensorische Auffälligkeiten

Auffällige Reaktion auf Annäherung anderer Kinder

Eingeschränktes Fantasiespiel

Eingeschränktes teilen

Eingeschränkte Koordination von Zeigegeste und Blickkontakt

Sprachliches Mitteilen v. a. überwiegend im Kontext von Bedürfnissen und Information

ses Schrittes ist v.a. bei jungen Kindern der Kinderarzt entscheidend.

Eine multidimensionale und multiprofessionelle Entwicklungsabklärung bildet die Basis einer spezialisierten Diagnostik (• Abb. 3). Je nach Lebensalter und Entwicklungsstand können standardisierte Fragebogen die Anamnese unterstützen. Ab einem Alter von 2 Jahren kann das „Diagnostische Interview für Autismus" in seiner revidierten Fassung (ADI-R) verwendet werden [6].

Neben der klinischen Verhaltensbeobachtung ist eine standardisierte Verhaltensbeobachtung (ADOS-2; Autism Diagnostic Observation Schedule; gegliedert in 5 Module mit Einsetzbarkeit $\mathrm{ab}$ dem 12. Monat bis ins Erwachsenenalter) sinnvoll [6].

Sowohl ADOS-2 als auch ADI-R zeigen in den Händen erfahrener Untersucher eine gute Sensitivität, aber eine geringe Spezifität. Auffällige Ergebnisse
Differenzialdiagnosen und begleitende Störungen

Eine Reihe von Störungsbildern findet sich in einer erhöhten Rate bei ASS und kommt sowohl als Differenzialdiagnose als auch als Komorbidität in Betracht (- Tab. 3). Angesichts dieser Problematik kann es teilweise sinnvoll sein, vor der diagnostischen Festlegung ein symptomatisch-therapeutisches Vorgehen $\mathrm{zu}$ wählen.

\section{Kognitive Beeinträchtigung}

Im Rahmen der ASS-Abklärung ist die Beurteilung der kognitiven Funktionen, welchen ja auch eine erhebliche prognostische Bedeutung zukommt, elementar [14].

30-40\% der ASS-Betroffenen haben eine Intelligenzminderung, 14-24\% eine unterdurchschnittliche Intelligenz. Hochbegabung (3\%) und außergewöhnliche Inselbegabungen (z.B.: „savants") sind selten $[6,15]$.

Bei schwerer kognitiver Beeinträchtigung mit kognitivem Referenzalter $<12$ bis 14 Monaten ist eine ASS-Diagnose nicht möglich [16].

\section{Sprachstörungen}

Die formale Sprachentwicklung ist bei $>60 \%$ der von ASS betroffenen Kindern beeinträchtigt und bleibt bei ca. zwei Dritteln bis zum 3. Lebensjahr im Wesentlichen aus. Bis zu ein Drittel autistischer Kinder erwirbt im Lebenszeitverlauf keine verbale Kommunikation [12, 14].

Im DSM-5 wird eine „soziale Kommunikationsstörung“ als eigene Kategorie definiert, welche sich durch Fehlen von restriktiven und repetitiven Verhaltensweisen oder Interessen von ASS abgrenzt (• Tab. 1).

Differenzialdiagnostisch stellen Sprachentwicklungsstörungen mit schwerer Einschränkung der rezeptiven Sprache eine Herausforderung dar. Betroffene Kinder verfügen in aller Regel über ein entwicklungsadäquates Repertoire gestischer und nonverbaler Kommunikationsmöglichkeiten, welches sie zur Interaktion in wechselseitiger Weise nutzen. 


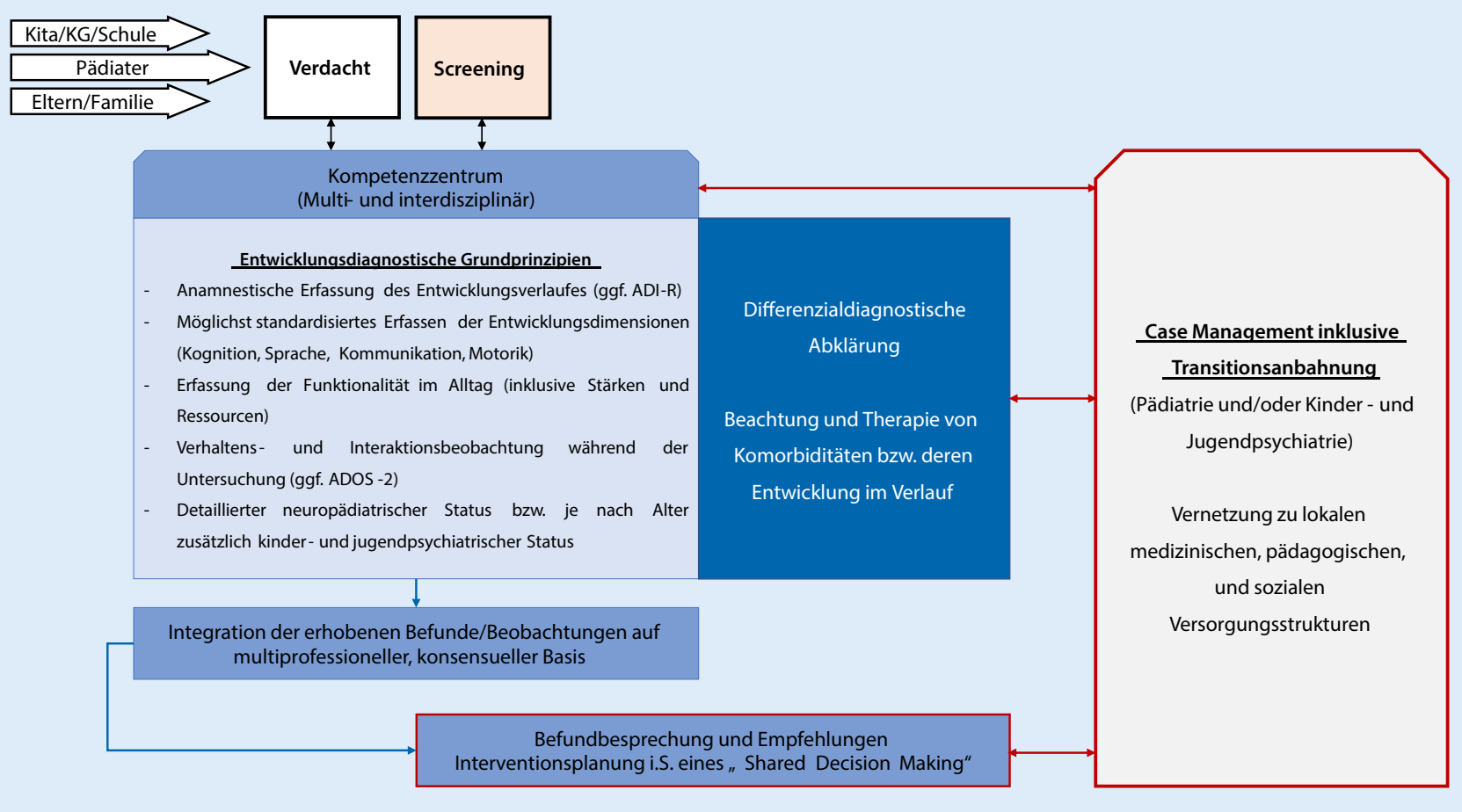

Abb. 3 \ Schematische Darstellung des diagnostischen Flow. ADI-R Diagnostisches Interview für Autismus - revidiert, ADOS Autism Diagnostic Observation Schedule, KG Kindergarten, Kita Kindertagesstätte

\section{Aufmerksamkeitsdefizit- und Hyperaktivitätssyndrom}

ADHS als Komorbidität liegt bei $28-43 \%$ der Kinder mit ASS vor [17]. Etwa ein Drittel der ADHS-Patienten zeigt autismusähnliche Symptome wie Einschränkung der exekutiven Funktionen mit Emotionsdysregulation, eingeschränkte Rollenübernahmefähigkeit, oft flüchtig wirkendem Blickkontakt und einer v. a. auch in sozialen Kontexten wirksam werdenden Unaufmerksamkeit. Wichtiges Unterscheidungsmerkmal sind die Reziprozität, die nonverbale Kommunikation, der Gestengebrauch, das oft fantasievolle Spielverhalten und das prinzipielle Interesse an Beziehungen zu anderen.

\section{ASS-ähnliche Symptome bei Kindern mit Deprivation}

Seit Bowlbys WHO-Report [18] bezüglich institutioneller frühkindlicher Deprivation sind die schweren neurobiologischen, sozialen, verhaltensbezogenen und kognitiven Folgen, u.a. im Sinne einer schweren reaktiven Bindungsstörung, unbestritten. Waisenkinder mit $>6$ Monaten Institutionalisierung zeig- ten als junge Erwachsene in $20 \%$ der Fälle autismusähnliche Symptome, welche unter dem Begriff „Quasi-Autismus“ ihren noch nicht in Klassifikationen dargestellten Ausdruck finden [19]. Deprivationsphänomene zeigen sich v.a. in jungen Jahren bei adäquater Intervention als reversibel.

In diesem Kontext erscheint auch der frühe und intensive Konsum elektronischer Medien (z. B.: TV, YouTube-Kanäle, PC) auf Kosten direkter elterlicher $\mathrm{Zu}$ wendung ein entwicklungsgefährdender Faktor zu sein [20].

ASS-Symptome im Kontext von Sinnesbeeinträchtigungen sind eine differenzialdiagnostische Herausforderung. Die Prävalenz von ASS bei Personen mit Hörstörung, Sehstörung oder dualer Sinnesbeeinträchtigung ist aufgrund unzureichender diagnostischer Methoden uneindeutig. Bei Taubblinden zeigt sich, ebenso wie für Menschen mit schwerer Intelligenzminderung, dass stereotype Muster alleine keine ASS-Diagnose begründen können. Differenzierende Merkmale sind alleine die soziale Aufmerksamkeit, Zuwendungsfähigkeit und emotionale Wechselseitigkeit [21].

\section{Emotionale und affektive Störungen}

Im Hinblick auf emotional-affektive Störungen kommt den mutistischen Störungen und den Sozialphobien eine besondere differenzialdiagnostische Bedeutung $\mathrm{zu}[22]$.

\section{ASS-Frühintervention und Begleitung}

Strukturierte, programmbasierte Intervention mit frühem Beginn $(2-4 \mathrm{LJ})$ ist effektiv [7]. ASS-spezifische Frühintervention scheint sich auch auf neurobiologischer Ebene auszuwirken. Veränderung der zerebralen Konnektivität und Normalisierung der EEG-Aktivität wurden gezeigt [23].

\section{Frühintervention}

Strukturierte Elternanleitungsprogramme (elternvermittelte Intervention) zur Stärkung der Eltern-Kind-Interaktion auf Basis eines sensitiven Erkennens von Möglichkeiten, um Aufmerksamkeit und Wechselseitigkeit in Spiel und Alltag zu vermitteln, zeigten signifikante Effekte 
Tab. 3 Gegenüberstellung wichtiger Komorbiditäten und Differenzialdiagnosen in Anlehnung an die AWMF-Leitlinie [6, 7]

\begin{tabular}{|c|c|}
\hline Komorbiditäten & Differenzialdiagnosen \\
\hline $\begin{array}{l}\text { Entwicklungsstörungen } \\
\text { Globale Entwicklungsverzögerung/ } \\
\text { Intelligenzminderung } \\
\text { Sprachstörungen } \\
\text { Lernstörungen } \\
\text { Umschriebene Entwicklungsstörung der motorischen } \\
\text { Funktion }\end{array}$ & $\begin{array}{l}\text { Entwicklungsstörungen } \\
\text { Globale Entwicklungsverzögerung/ } \\
\text { Intelligenzminderung } \\
\text { Sprachstörungen } \\
\text { Lernstörungen }\end{array}$ \\
\hline $\begin{array}{l}\text { Psychische oder Verhaltensprobleme oder Störungen } \\
\text { ADHS } \\
\text { Angststörungen/Phobien (inkl. sozialer Phobie) } \\
\text { Affektive Störungen } \\
\text { Zwangsstörungen } \\
\text { Bindungsstörungen } \\
\text { Schlafstörungen } \\
\text { Essstörungen (Pica, Adipositas, vermeidend/restriktiv } \\
\text { [ARFID]) }\end{array}$ & $\begin{array}{l}\text { Psychische oder Verhaltensprobleme } \\
\text { oder Störungen } \\
\text { ADHS } \\
\text { Angststörungen/Phobien (inkl. sozialer } \\
\text { Phobie) } \\
\text { Affektive Störungen } \\
\text { Zwangsstörungen } \\
\text { Bindungsstörungen } \\
\text { Psychosen } \\
\text { Mutismus }\end{array}$ \\
\hline $\begin{array}{l}\text { Körperlich-neurologische Störungen } \\
\text { Epilepsie } \\
\text { Enzephalopathien } \\
\text { Genetische Syndrome } \\
\text { Sinnesbeeinträchtigungen }\end{array}$ & $\begin{array}{l}\text { Körperlich-neurologische Störungen } \\
\text { Enzephalopathien (v. a. auch epileptisch) } \\
\text { Genetische Syndrome } \\
\text { Sinnesbeeinträchtigungen } \\
\text { Stoffwechselstörungen }\end{array}$ \\
\hline $\begin{array}{l}\text { Funktionelle Probleme und Störungen } \\
\text { Harninkontinenz/Enuresis } \\
\text { Obstipation } \\
\text { Stuhlinkontinenz/Enkopresis } \\
\text { Schlafstörungen }\end{array}$ & - \\
\hline ARFID „avoidant restrictive food intake disorder" (DSM-5-D & iagnose) \\
\hline
\end{tabular}

auf die soziale Kommunikation der ASSbetroffenen Kinder [24, 25].

In patientenzentrierten, verhaltenstherapeutischen Interventionen werden Spielangebote und Möglichkeiten zur sozialen Interaktion mit Orientierung am natürlichen Entwicklungsverlauf gesetzt.

Diese therapeutenvermittelten Programme arbeiten meist im Eins-zu-EinsSetting mit hoher Intensität (15-20 h/ Woche). Signifikante Verbesserungen mit mittleren Effektstärken für kognitive und sprachliche Entwicklungsfortschritte wurden gezeigt [24].

Verschiedene evidenzbasierte Programme inkludieren Elternarbeit und direkt therapeutenvermittelte Intervention (z.B. Joint Attention, Symbolic Engagement and Regulation [JASPER] oder Early Start Denver Model [ESDM], [7]).

Im deutschsprachigen Raum besteht bezüglich strukturierter, programmbasierter Frühintervention ein versorgungsrelevantes Defizit. Evaluierte Adaptierungen z.B. des ESDM sind aber zunehmend beobachtbar [26].
Unterstützende, das Umfeld und Zeitabläufe strukturierende Konzepte (z.B. TEACCH [treatment and education of autistic and communication handicaped children and adults], [27]) helfen, den Alltag in allen Lebensphasen zu stabilisieren.

\section{Begleitung im Schulalter und im frühen Erwachsenenalter}

Mit zunehmendem Lebensalter gewinnen Beziehungen zu Gleichaltrigen an Bedeutung, was oftmals zur Überforderung von Kindern mit ASS führt und sich in schwierigen Verhaltensmustern niederschlagen kann.

Der Einsatz von Bildgeschichten, z. B. im Sinne des Ansatzes der „social stories“, ist hilfreich, um auf zu erwartende Ereignisse vorzubereiten oder um durch Erklärung alltäglicher Abläufe soziales Verständnis zu fördern (• Abb. 5; [28]).

Eine gute Einbindung der PädagogInnen und Aufklärung der Mitschüler sind wichtig, um die Entwicklung von unnötigen Traumatisierungen für betroffene
Kinder hintanzuhalten und um entwicklungsfördernde Voraussetzungen zu etablieren.

Verbesserungen im Sozialverhalten bei Kindern im späten Vorschul- und Schulalter können durch gut strukturierte soziale Kompetenzgruppen erreicht werden [14].

Die sich oft mit zunehmendem Lebensalter verstärkt manifestierenden psychischen Probleme führen oft zur Behandlung mit Psychopharmaka [14] und spielen eine wesentliche Rolle bezüglich des Gelingens der Integration ins Arbeitsleben und der selbstständigen Lebensgestaltung.

Nur ein Viertel der von ASS betroffenen Erwachsenen mit durchschnittlicher Intelligenz lebt im eigenen Haushalt. Lebenspartnerschaften sind deutlich seltener als in der Allgemeinbevölkerung [7, 14].

Bei Jugendlichen mit zusätzlichen intellektuellen Einschränkungen stellt sich die Frage nach rechtlichen Vertretungsmöglichkeiten und Lebensformen mit dauerhafter Begleitung.

\section{Therapie}

Eine direkt die ASS-Kernsymptome adressierende medikamentöse Therapie ist derzeit nicht möglich. Sind verhaltensanalytische (z.B. ABC-Schema) und verhaltenstherapeutische Möglichkeiten ausgeschöpft, kann eine symptomorientierte medikamentöse Behandlung sinnvoll werden (- Tab. 4). Grundprinzipien der medikamentösen Therapie sind hier v. a. klare Definition des Therapiezieles und der angedachten Therapiedauer, vorsichtige Aufdosierung und regelmäBige Überprüfung auf Wirksamkeit und Nebenwirkungen.

Diätetische Maßnahmen, darunter auch eine glutenfreie und kaseinfreie Diät, sind nicht ausreichend evidenzbasiert, um generell empfohlen werden zu können [7, 14]. Ähnliches gilt für viele weitere nichtevidenzbasierte Therapieansätze (z.B.: hyperbare Sauerstofftherapie, unterschiedliche Vitaminsubstitutionen etc.). Mit Familien, die sich dennoch für derartige Therapieansätze entscheiden, ist es wichtig, in 


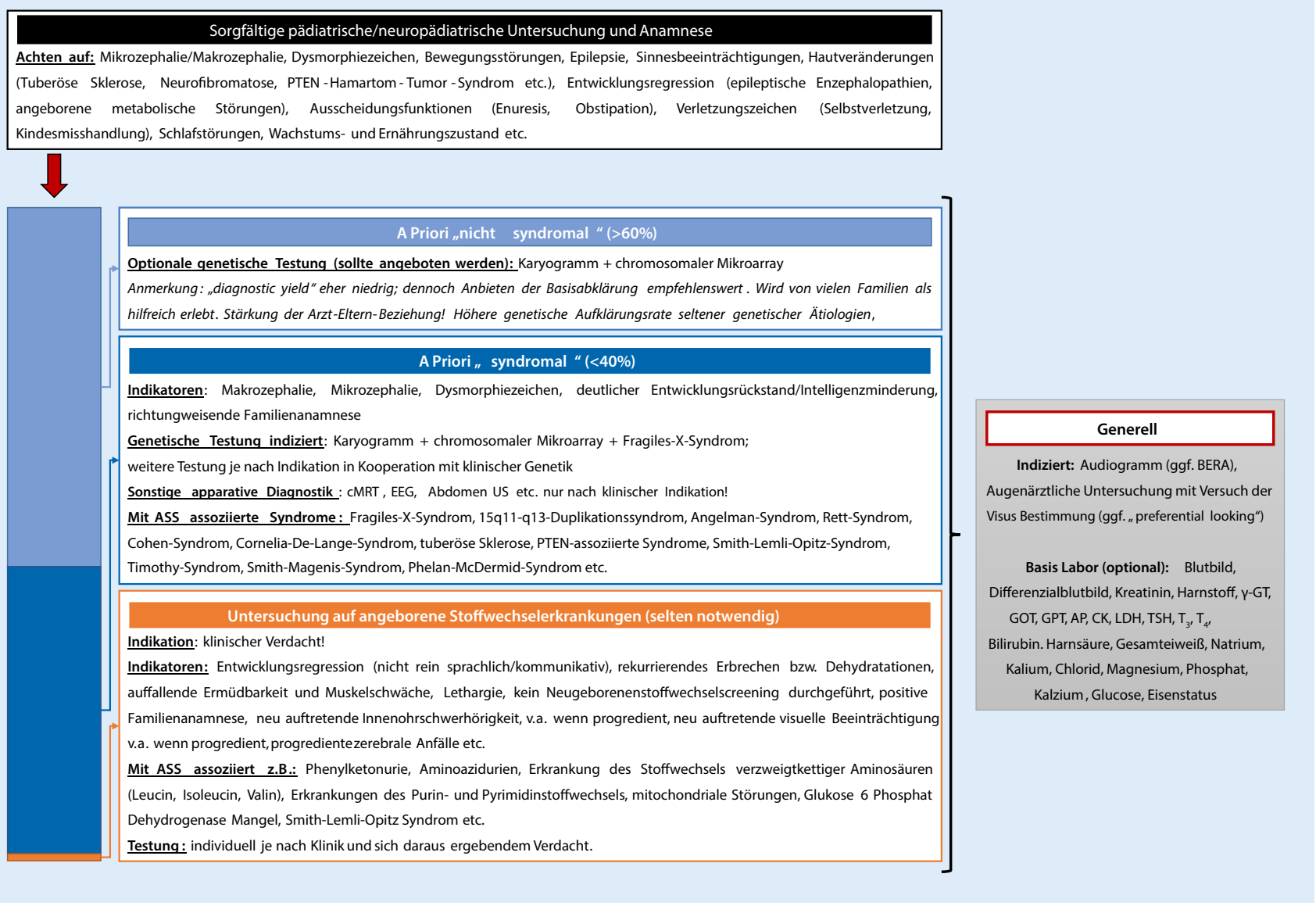

Abb. $4 \Delta$ Medizinische Abklärung nach ASS-Diagnose

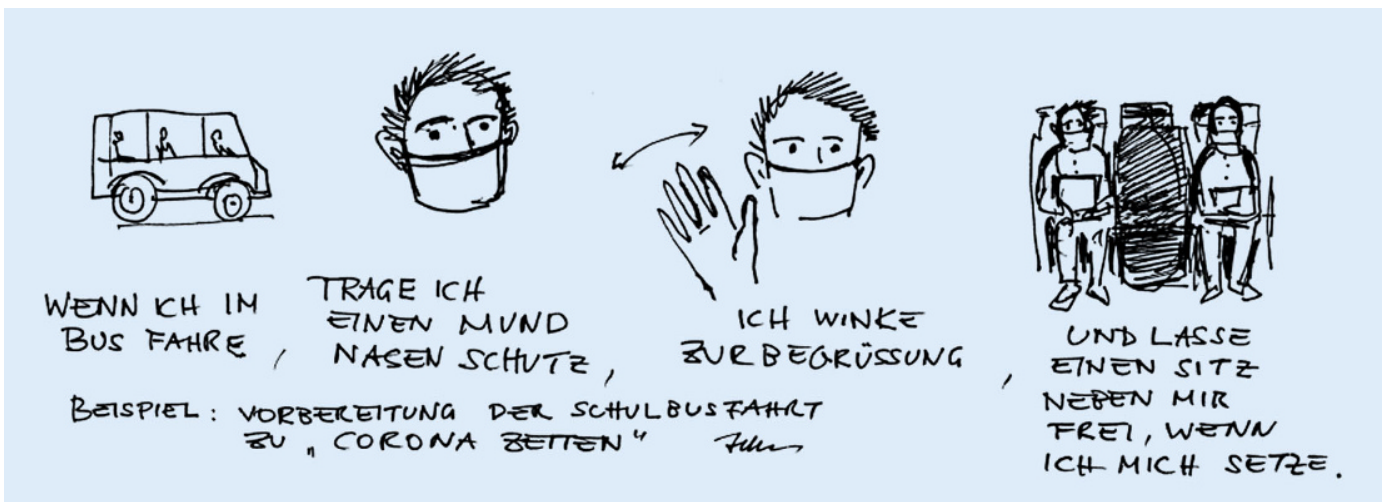

Abb. $5<$ Beispiel einer einfachen visuellen Orientierungshilfe

respektvollem Kontakt zu bleiben, um ggf. Sekundärschäden zu vermeiden.

\section{Arbeit mit Familien}

Ein Kind mit Autismus prägt die Entwicklung einer gesamten Familie. Eltern berichten von höherer Stressbelastung und wirtschaftlichen Herausforderungen.
Es ist daher von großer Bedeutung, dass Kinderärzte/Kinderärztinnen oder Kinder- und Jugendpsychiater/-innen diesen Familien eine sozialmedizinische „Heimat“ bieten. Diese Heimat ist durchaus in Anlehnung an das Konzept des „medical home“ zu verstehen [29]. Dieses Modell geht von einer Partnerschaft zwischen Arzt/Ärztin und Familie aus, die sich u. a. in einer Kultur des gemeinsamen Entscheidungssuchens und -treffens („shared decision-making“) ausdrückt.

Ein weiteres Element des Medical home ist die Vernetzungsarbeit hin $\mathrm{zu}$ Selbsthilfeorganisationen und Einrichtungen der Sozialhilfe und des Bildungssystems. Dies ist umso bedeutungsvoller, wenn es gilt, Jugendliche mit ASS beim Gelingen des Übergangs ins Erwachsenenalter zu unterstützen. 
Tab. 4 Exemplarische Auflistung möglicher Medikationsindikationen und ggf. geeigneter Medikamentengruppen. (Hyman et al. [14])

\section{(Beispiele) \\ SNRI (Atomoxetin) \\ $a_{2}$-Agonisten (Gu- \\ anfacin, Clonidin)}

Substanzgruppe Zielsymptome

Stimulanzien (Me- Hyperaktivität Klare Definition des Therapiezieles

thylphenidat, Am- Impulsivität Vorsichtige Aufdosierung

phetaminsulfat, Unaufmerksamkeit Regelmäßige Überprüfung auf Wirksamkeit und NebenLisdexamfetamin) Ablenkbarkeit wirkungen

Atypische Neuro- Irritabilität leptika (Aripiprazol, Aggressivität Risperidon)

SSRI (Fluoxetin, Sertralin)

\section{Melatonin} Niedrigpotente Neuroleptika

(Chlorprothixen,

Melperon, Pipamperon)

Antihistaminika

(Doxylamin)

Verhaltensintervention (ABC-Analyse) und Umfeldgestaltung (z. B.: TEACCH) ausgeschöpft?

Klare Definition des Therapiezieles und der Therapiedauer

Wenn möglich, vorsichtige Aufdosierung

Regelmäßige Überprüfung auf Wirksamkeit und Nebenwirkungen

Cave: Gewichtszunahme, extrapyramidale Nebenwirkungen, Sedierung

Ängstlichkeit V.a. in Bezug auf Ängstlichkeit genaue Analyse notwenDepression dig

Zwänge Regelmäßige Überprüfung auf Wirksamkeit und Nebenwirkungen

Schlafstörungen Primäre Bausteine: Maßnahmen der Psychoedukation, der Schlafhygiene sowie verhaltenstherapeutische Maßnahmen

Häufig bei Kindern nicht zugelassen (individueller Heilversuch!)

Wahl der Substanz richtet sich nach der Form der Schlafstörung

Bei Einschlafstörungen und Tag-Nacht-Rhythmusstörungen stellt Melatonin eine gut verträgliche Option dar

Führen eines Schlafprotokolls

Regelmäßige Überprüfung auf Wirksamkeit und Nebenwirkungen

$A B C_{\text {"I }}$ antecedents/behaviour/consequences", SNRI Serotonin-Noradrenalin-Wiederaufnahmehemmer, SSRI selektive Serotonin-Wiederaufnahmehemmer, TEACCH Treatment and Education of Autistic and related Communication Handicapped Children

Die Rolle des Medical home ist weniger mit einem spezifischen Setting als vielmehr an ärztliche Persönlichkeiten geknüpft, die Freude daran haben, mit Familien den Entwicklungsweg zu gehen und dabei dem Kind und den Eltern ihre Stärken bewusst zu machen, das Erreichen von Meilensteinen mitzufeiern und in Krisen Anlaufstelle zu sein, ohne die Familie zu bevormunden.

\section{Fazit für die Praxis}

\section{- Autismus-Spektrum-Störungen}

(ASS) zeigen eine Prävalenz von

$1 \%$ und gehen mit einer breiten

Phänotypologie einher.

- Früherkennung ist häufig bereits im

2. Lebensjahr möglich. Eine Schlüssel-
Hier steht eine Anzeige. 


\section{- Angesichts der hohen Belastung für Familie und Umfeld ist ein kompe- tentes ärztliches Case Management mit Vernetzung zu Systempartnern aus Bildungs- und Sozialwesen not- wendig.}

\section{Korrespondenzadresse}

Johannes Fellinger
Institut für Sinnes und Sprachneurologie,
Konventhospital Barmherzige Brüder Linz
Bischofstraße 11, 4021 Linz, Österreich
johannes.fellinger@bblinz.at

Funding. Open access funding provided by Johannes Kepler University Linz.

\section{Einhaltung ethischer Richtlinien}

Interessenkonflikt. J. Hofer und J. Fellinger geben an, dass kein Interessenkonflikt besteht.

Für diesen Beitrag wurden von den Autoren keine Studien an Menschen oder Tieren durchgeführt. Für die aufgeführten Studien gelten die jeweils dort angegebenen ethischen Richtlinien.

Open Access. Dieser Artikel wird unter der Creative Commons Namensnennung 4.0 International Lizenz veröffentlicht, welche die Nutzung, Vervielfältigung, Bearbeitung, Verbreitung und Wiedergabe in jeglichem Medium und Format erlaubt, sofern Sie den/die ursprünglichen Autor(en) und die Quelle ordnungsgemäß nennen, einen Link zur Creative Commons Lizenz beifügen und angeben, ob Änderungen vorgenommen wurden.

Die in diesem Artikel enthaltenen Bilder und sonstiges Drittmaterial unterliegen ebenfalls der genannten Creative Commons Lizenz, sofern sich aus der Abbildungslegende nichts anderes ergibt. Sofern das betreffende Material nicht unter der genannten Creative Commons Lizenz steht und die betreffende Handlung nicht nach gesetzlichen Vorschriften erlaubt ist, ist für die oben aufgeführten Weiterverwendungen des Materials die Einwilligung des jeweiligen Rechteinhabers einzuholen.

Weitere Details zur Lizenz entnehmen Sie bitte der Lizenzinformation auf http://creativecommons.org/ licenses/by/4.0/deed.de.

\section{Literatur}

1. Xu G, Strathearn L, Liu B et al (2018) Prevalence of autism spectrum disorder among US children and adolescents, 2014-2016. JAMA319(1):81-82

2. Idring S, Rai D, Dal H et al (2012) Autism spectrum disorders in the Stockholm Youth Cohort: design, prevalence and validity. Plos One 7(7):e41280

3. Palmer N, Beam A, Agniel Det al (2017) Association of sex with recurrence of autism spectrum disorder among siblings. JAMA Pediatr 171(11):1107-1112
4. Tick B, Bolton $P$, Happé $F$ et al (2016) Heritability of autism spectrum disorders: a meta-analysis of twin studies. J Child Psychol Psychiatry Allied Discip 57(5):585-595

5. Myers SM, Challman TD, Bernier R et al (2020) Insufficient evidence for "autism-specific" genes. Am JHum Genet 106(5):587-595

6. Freitag C, Vllasaliu L (2016) Autismus-SpektrumStörungen im Kindes-, Jugend- und Erwachsenenalter Teil 1:Diagnostik. S3-Leitlinie

7. Lord C, Elsabbagh M, Baird G et al (2018) Autism spectrum disorder. Lancet 392(10146):508-520

8. Lord C, Jones RM (2012) Annual research review: re-thinking the classification of autism spectrum disorders. J Child Psychol Psychiatry Allied Discip 53(5):490-509

9. Pearson N, Charman T, Happé F et al (2018) Regression in autism spectrum disorder: reconciling findings from retrospective and prospective research. Autism Res 11(12):1602-1620

10. Pickles A, Anderson DK, Lord C (2014) Heterogeneity and plasticity in the development of language: a 17-year follow-up of children referred early for possible autism. J Child Psychol Psychiatry Allied Discip 55(12):1354-1362

11. Pugliese CE, Anthony L, Strang JF et al (2015) Increasing adaptive behavior skill deficits from childhood to adolescence in autism spectrum disorder: role of executive function. J Autism Dev Disord 45(6):1579-1587

12. Baghdadli A, Assouline B, Sonié S et al (2012) Developmental trajectories of adaptive behaviors from early childhood to adolescence in a cohort of 152 children with autism spectrum disorders. J Autism Dev Disord 42(7):1314-1325

13. Robins DL, Casagrande K, Barton M et al (2014) Validation of the modified checklist for Autism in toddlers, revised with follow-up (M-CHAT-R/F) Pediatrics 133(1):37-45

14. Hyman SL, Levy SE, Myers SM (2020) Identification, evaluation, and management of children with autism spectrum disorder. Pediatrics 145(1):e20193447

15. Baio J, Wiggins L, Christensen DL et al (2018) Prevalence of Autism Spectrum Disorder Among Children Aged 8 Years-Autism and Developmen tal Disabilities Monitoring Network. Morb Mortal 67(6):1-23

16. Pierce K, Gazestani VH, Bacon E et al (2019) Evaluation of the diagnostic stability of the early autism spectrum disorder phenotype in the general population starting at 12 months. JAMA Pediatr 173(6):578-587

17. Simonoff E, Pickles A, Charman T et al (2008) Psychiatric disorders in children with autism spectrum disorders: prevalence, comorbidity, and associated factors in a population-derived sample. J Am Acad Child Adolesc Psychiatry 47(8):921-929

18. Bowlby J (1952) Maternal Care and Mental Health: A Report Prepared on Behalf of the World Health Organization as a Contribution to the United Nations Programme for the Welfare of Homeless Children, 2NDed. Monograph series/World Health Organization, no. 2. Organization

19. Sonuga-Barke EJS, Kennedy M, Kumsta R et al (2017) Child-to-adult neurodevelopmental and mental health trajectories after early life deprivation: the young adult follow-up of the longitudinal English and Romanian Adoptees study. Lancet 389(10078):1539-1548

20. Heffler KF, Sienko DM, Subedi K et al (2020) Association of early-life social and digital media experiences with development of autism spectrum disorder-like symptoms. JAMA Pediatr 174(7):690-696

21. Hoevenaars-van den Boom MAA, Antonissen ACFM, Knoors $\mathrm{H}$ et al (2009) Differentiating characteristics of deafblindness and autism in people with congenital deafblindness and profound intellectual disability. J Intellect Disabil Res 53(6):548-558

22. Kamp-Becker I, Stroth S, Stehr T (2020) AutismusSpektrum-Störungen im Kindes- und Erwachsenenalter: Diagnose und Differenzialdiagnosen (Autism spectrum disorder in childhood and adults: diagnosis and differential diagnoses). Nervenarzt 91(5):457-470

23. Dawson G, Jones EJH, Merkle K et al (2012) Early behavioral intervention is associated with normalized brain activity in young children with autism. J Am Acad Child Adolesc Psychiatry 51(11):1150-1159

24. Weitlauf AS, McPheeters ML, Peters B et al (2014) Therapies for Children With Autism Spectrum Disorder: Behavioral Interventions Update. Rockville, MD

25. Pickles A, Le Couteur A, Leadbitter K et al (2016) Parent-mediated social communication therapy for young children with autism (PACT): long-term follow-up of a randomised controlled trial. Lancet 388(10059):2501-2509

26. Holzinger D, Laister D, Vivanti G et al (2019) Feasibility and outcomes of the early start denver model implemented with low intensity in a community setting in Austria.J Dev Behav Pediat 40(5):354-363

27. Virues-Ortega J, Julio FM, Pastor-Barriuso R (2013) The TEACCH program for children and adults with autism: a meta-analysis of intervention studies. Clin Psychol Rev 33(8):940-953

28. Gray C, Prizant BM (2015) The new social story book. Pre-school children, young adults: over 180 social stories: that teach everyday social skills to children and young adults with autism or asperger's sydrome, and their peers, 15th anniversary Edition. Future Horizons, Arlington

29. Carbone VJ, Sweeney-Kerwin EJ, Attanasio V et al (2010) Increasing the vocal responses of children with autism and developmental disabilities using manual sign mand training and prompt delay. JAppl Behav Anal 43(4):705-709

30. Venker CE, Ray-Subramanian CE, Bolt DM et a (2014) Trajectories of autism severity in early childhood. J Autism Dev Disord 44(3):546-563

31. Kim SH, Bal VH, Benrey N et al (2018) Variability in Autism Symptom Trajectories Using Repeated Observations From 14 to 36 Months of Age. J Am Acad Child Adolesc Psychiatry 57(11):837-848.e2 